\section{Questión}

Periodismo / Comunicación

ISSN 1669-6581

La cuarta revolución industrial. Una visión economicista del cambio social Marcelo Norberto Botto

Question/Cuestión, Vol. 2, № 66, Agosto 2020

ISSNe 1669-6581

https://perio.unlp.edu.ar/ojs/index.php/question/index

IICom-FPyCS-UNLP

DOI: https//doi.org/10.24215/16696581e461

\title{
La cuarta revolución industrial
} una visión economicista del cambio social

The fourth industrial revolution an economic vision of social change

Marcelo Norberto Botto

Facultad de Comunicación Universidad Argentina de la Empresa

Argentina

marcelobotto@yahoo.com.ar http://orcid.org/0000-0003-3693-7068 


\title{
Resumen
}

Uno de los campos de las ciencias sociales que concibió históricamente a la tecnología como una herramienta crucial para el progreso y la transformación de las sociedades es la Economía. Desde esta visión autores como Machlup, Bell, Masuda, Castells y Schwab explicaron las transformaciones sociales a partir de la segunda mitad del siglo XX hasta la actualidad mediante la relación economía-tecnología. Desde esta perspectiva la producción de conocimiento es entendida como una actividad económica derivada de la capacidad de la sociedad de crear tecnologías aplicadas al desarrollo social.

\section{Palabras clave}

Sociedad del conocimiento, Comunicación, Cuarta revolución industrial, Tecnología, Economía.

\begin{abstract}
One of the fields of social sciences that historically conceived of technology as a crucial tool for the progress and transformation of societies is the Economy. From this vision, authors such as Machlup, Bell, Masuda, Castells and Schwab explained the social transformations from the second half of the 20th century to the present through the economy-technology relationship. From this perspective, the production of knowledge is understood as an economic activity derived from the ability of society to create technologies applied to social development.
\end{abstract}




\section{Key Words}

Knowledge society, Communication, Fourth industrial revolution, Technology, Economy.

\section{Introducción}

Las transformaciones que las tecnologías impulsan en los sistemas sociales fueron objeto de análisis por parte de teóricos de variadas disciplinas desde que la visión positivista postuló el progreso constante de la sociedad debido a la capacidad racional del ser humano para dominar a la naturaleza y transformar su entorno. Las tecnologías, desde ese momento, fueron percibidas como herramientas clave creadas por la inventiva humana para ser utilizadas con esas finalidades.

El debate, a partir de entonces, giró en torno a la creatividad de los individuos para modelar tecnologías que son utilizadas para la transformación de las sociedades y, desde otra perspectiva, el poder de las tecnologías para generar por sí mismas cambios sociales.

Uno de los campos de las ciencias sociales que concibió históricamente a la tecnología como una herramienta crucial para el progreso y la transformación de las sociedades es la Economía.

El sociólogo estadounidense Daniel Bell da cuenta de esta relación al asegurar que «la tecnología es el fundamento de la sociedad industrial. La innovación y la transformación económica dependen directamente de la nueva tecnología» (1991, p. 222).

Los autores que abordan esta temática coinciden en señalar que la relación tecnología-economía se fue fortaleciendo con el paso del tiempo. Tuvo origen 
con las explicaciones vinculadas con el desarrollo evolutivo de las sociedades en la que las aplicaciones tecnológicas estaban asociadas al esfuerzo humano con un predominio de la actividad agrícola, a la que le siguió una segunda etapa con el advenimiento de la primera revolución industrial (1770-1840) en la que el esfuerzo mecánico corporizado por la construcción del ferrocarril y el motor a vapor sustituyó al trabajo humano, mientras que la segunda revolución industrial -durante el último tercio del siglo XIX y comienzos del XX- hizo posible la producción en masa, fomentada por el desarrollo de la tecnología eléctrica y la cadena de montaje lo que dio origen a la denominada sociedad de masas.

Un tipo de sistema social basado en la producción industrial cobró cada vez mayor impulso a lo largo del siglo XX pero, a partir de la segunda mitad de esa centuria, fue experimentando cambios estructurales que fueron percibidos $y$ explicados de distinta manera.

Autores como Fritz Machlup -al que se le atribuye el origen del concepto Sociedad de la información y del conocimiento- Alain Touraine, Daniel Bell, Alvin Toffler, Yoneji Masuda, Manuel Castells, entre otros, esbozaron explicaciones sobre el impacto positivo que arrojó el uso de los adelantos tecnológicos como motor de desarrollo de las naciones a causa del impulso de la producción y el empleo.

Ya en el siglo XXI el economista alemán, Klaus Schwab asegura que la tecnología y la digitalización son las impulsoras de una nueva revolución sin precedentes en la historia de la humanidad por su velocidad, magnitud y alcance.

Desde una visión que pone el foco principalmente en los factores asociados al crecimiento económico, el presidente del Foro Económico Mundial sostiene 
que, en los albores del siglo XXI, las nuevas tecnologías desencadenan transformaciones profundas en los sistemas económicos y en las estructuras sociales.

Los cambios que percibe los asocia con la posibilidad de conexión entre miles de millones de personas a dispositivos móviles, que deriva en una capacidad de procesamiento y almacenamiento nunca antes alcanzado. Pero además, por los acelerados avances tecnológicos en los campos de la inteligencia artificial, la robótica, internet de las cosas, los vehículos autónomos, la impresión 3D, la nanotecnología, la biotecnología, la ciencia de materiales, el almacenamiento de energía y la computación cuántica, entre otros (Schwab, 2016, p. 8).

Los factores mencionados son la base de un cambio que visualiza en marcha y que denomina Cuarta revolución industrial.

\section{Hacia una sociedad del conocimiento}

La idea de que la productividad económica se encuentra directamente asociada al desarrollo de nuevas tecnologías, las que permitirían al hombre hacer menos y pensar más estuvo en la mente de una buena cantidad de economistas que predijeron que la economía sería sustentada en la información y la creación de conocimiento.

Desde esta perspectiva la producción de conocimiento es entendida como una actividad económica derivada de la capacidad de la sociedad de crear tecnologías aplicadas al desarrollo social.

En tal sentido, el economista austríaco Machlup, establece esta relación al estudiar la producción desarrollada por los sectores gubernamentales, empresariales e industriales estadounidenses hacia fines de la década de 
1950 y los beneficios sociales derivados de la transformación de la producción y el empleo.

Para ello analizó los resultados de encuestas difundidas por distintos organismos estadounidenses sobre más de treinta ramas de la producción -que el autor denomina industrias productoras de conocimiento- y las relacionó con el valor de su producto, lo que le permitió formar una amplia base de datos cuantitativa para dar cuenta del papel de la producción de conocimiento en el producto bruto nacional del país del norte.

En consecuencia, señaló que la producción del conocimiento está vinculada con el desarrollo tecnológico, lo que trae cambios en los tipos de ocupaciones laborales:

Si bien el ascenso de las ocupaciones productoras de conocimiento forma parte de un proceso ininterrumpido, ha habido una sucesión de ocupaciones liderando este movimiento, primero administrativo, luego administrativo y gerencial, y ahora del personal profesional y técnico. Así, el cambio de patrón de empleo indica un movimiento continuo de las tareas manuales a mentales, y de menos a más mano de obra altamente entrenada (Machlup, 1962, p. 396).

Este planteo que el economista expuso en su libro La producción y distribución del conocimiento en los Estados Unidos (1962) hizo que algunos autores le atribuyeran el origen del concepto Sociedad de la información y del conocimiento.

Touraine, desde una visión sociológica, y Bell, desde una mirada derivada de la economía, fueron los que presentaron con mayor fuerza -en los albores de la década de la 1970- la idea de que la sociedad industrial dejaba paso a un nuevo tipo de organización en occidente a la que el teórico estadounidense 
denominó Sociedad del conocimiento sustentada en tecnología de la información.

Ambos teóricos coinciden en que los países desarrollados presentan cambios en el sistema ocupacional que impulsan transformaciones de relevancia en la estructura social. Por una parte, perciben un declive en la demanda de empleados en los sectores industriales como las fábricas, mientras que por otro observan un aumento en la demanda de empleados con preparación profesional o técnica, en especial aquellos destinados a la producción de bienes intangibles, vinculado con el sector de los servicios.

Bell, por su parte, percibió a comienzos de la década de 1970 que el tipo de sociedad basada en la industrialización estaba experimentando cambios sustanciales que comenzaban a impactar en la estructura social organizada en torno a la producción y a la maquinaria para la fabricación de bienes, y comenzaba a dar paso a un sistema post-industrial, basado en la generación de servicios.

Luego de la segunda guerra mundial y, principalmente hacia mediados de la década de 1950, se experimentó un cambio hacia el desarrollo marcado de los empleos profesionales y técnicos, que requerían tradicionalmente una educación universitaria.

Bell brinda el caso específico de los Estados Unidos que, entre 1960 y principios de la década de 1970, «la tasa de crecimiento de la clase profesional y técnica como un todo ha sido el doble de la tasa media de crecimiento de la fuerza de trabajo, la tasa de crecimiento de los científicos e ingenieros ha sido el triple de la tasa de la población trabajadora» (1991, p. 34). 
Esta transición entre el cambio de época la enfatiza a través del prefijo post, para destacar que los factores asociados a lo científico y tecnológico son las causas esenciales del advenimiento de un nuevo tipo de sociedad.

Bell explica por qué la denominación de sociedad post-industrial, en lugar de sociedad del conocimiento, sociedad de información o sociedad profesional. «El concepto de sociedad post-industrial subraya el carácter central del conocimiento teórico como eje a partir del cual se organizarán la nueva tecnología, el crecimiento económico y la estratificación de la sociedad» (Bell, 1991, p. 138).

Para el autor, en una sociedad post-industrial es importante la energía y adquiere valor la información, pero fundamentalmente se basa en los servicios -en la que queda relegada la fuerza del obrero, por lo que cada vez van a estar menos identificados con el trabajador de fábrica debido a que los trabajos mutaron hacia otros sectores-. En tal sentido, este entramado social requiere de profesionales educados y preparados para desarrollar las tareas que demanda los nuevos sectores de la economía.

De acuerdo a lo expuesto, una primera característica de la sociedad postindustrial es que la fuerza laboral se ocupa en los servicios de manera prioritaria, aunque el autor los diferencia de acuerdo a la siguiente clasificación. Servicios personales: tiendas minoristas, lavanderías, garajes, establecimientos de belleza; de negocios: bancos y financieras, inmobiliarias, seguros; de transporte, comunicación y servicios públicos; y sanidad, educación investigación y gobierno.

«Es el crecimiento de esa última categoría el decisivo en la sociedad postindustrial. $Y$ es esa categoría la que representa una nueva "inteligencia" en las 
universidades, las organizaciones de investigación, las profesiones y el gobierno» (Bell, 1991, p. 33).

Otra de las características de la sociedad post-industrial es que, particularmente en las naciones avanzadas, la porción de dinero destinado a la comida comienza a bajar y se deriva hacia la adquisición de bienes duraderos como ropa, casas, automóviles y para la diversión.

«De esta forma, un sector terciario, el de servicios personales, comienza a desarrollarse: restaurantes, hoteles, autoservicios, viajes, entretenimientos, deportes, al tiempo que los horizontes de la gente se expanden y se desarrollan nuevas necesidades y gustos» (Bell, 1991, p. 152).

Estos cambios operan sobre la conciencia de los individuos que aspiran a una mejor calidad de vida que se sustenta en dos áreas fundamentales: la salud y la educación.

La eliminación de la enfermedad y el creciente número de personas que pueden vivir una existencia plena, además de los esfuerzos para extender la duración de la vida, hacen de los servicios sanitarios un factor crucial de la sociedad moderna; y el desarrollo de las exigencias técnicas y la especialización profesional hace de la educación, y del acceso a la educación superior, la condición para entrar en la sociedad post-industrial. Así, nos hallamos ante el desarrollo de una nueva intelectualidad, en particular formada por los profesores (Bell, 1991, pp. 152 y 153).

La calidad de la vida, a partir de entonces, se mide por los servicios y comodidades en salud, educación, diversiones y las artes, que ahora son anhelos deseables y posibles para el conjunto social.

\section{La primacía del conocimiento teórico}


El conocimiento fue siempre necesario para el funcionamiento de todo tipo de sociedad. Lo que plantea el autor, en este caso, es que lo que caracteriza a la sociedad post-industrial es el cambio en el carácter del conocimiento.

En la sociedad post-industrial el carácter central del conocimiento es teórico lo que permite la innovación y el control del cambio para tratar de anticipar el futuro con el fin de planificarlo.

En este nuevo escenario adquiere fundamental importancia la relación entre la ciencia y la tecnología que se simboliza en la fase "investigación y desarrollo", lo que permitió el surgimiento de industrias basadas en la ciencia (computadoras, electrónica, óptica, polímeros) que dominan cada vez más el sector industrial de la sociedad y proporcionan la primacía a las sociedades industriales avanzadas (Bell, 1991, p. 43).

Es por esto que Bell asegura que el conocimiento teórico se convierte cada vez más en el recurso estratégico de una sociedad, por lo que las universidades, las organizaciones de investigación y las instituciones intelectuales son las estructuras centrales porque es donde se desarrolla el conocimiento teórico.

Y es del conocimiento teórico de donde surge una nueva tecnología intelectual, entendida por Bell como la sustitución de juicios intuitivos por algoritmos (normas para la solución de problemas) que se pueden incorporar en un programa de computadora para formalizar una serie de reglas de decisión (Bell, 1991, p. 48).

En tal sentido, un factor que resultó de suma importancia es el desarrollo y utilización de computadoras, debido a que facilitaron el puente entre la teoría formal y la acumulación de datos.

«Sin la computadora, las nuevas herramientas matemáticas habrían tenido sobre todo un interés intelectual con un bajo poder de resolución. La cadena de 
cálculos múltiples que se pueden hacer con facilidad, los análisis de muchas variables, la solución simultánea de centenares de ecuaciones solo son posibles con una herramienta de tecnología intelectual, la computadora» (Bell, 1991, p. 48).

Desde esta mirada la tecnología es percibida como una herramienta de sustancial importancia para la transformación social y Bell lo vincula con cinco factores definidos que motorizaron esos cambios:

1. Al producir más bienes a menor costo, la tecnología ha sido el motor fundamental de la elevación de los niveles de vida del mundo y de la reducción de las desigualdades en occidente

2. La tecnología ha creado la nueva clase, hasta ahora desconocida, del ingeniero y del técnico, hombres que se hallan separados del sitio de trabajo pero que constituyen un 'cuerpo de planificación' para las operaciones del proceso de trabajo.

3. La tecnología ha creado una nueva definición de racionalidad, una nueva forma de pensamiento, que pone de relieve las relaciones funcionales y las cuantitativas. Sus criterios de actuación son los de la eficiencia y la optimización.

4. Las revoluciones en el transporte y en la comunicación, como una consecuencia de la tecnología, han creado nuevas interdependencias económicas y nuevas interacciones sociales.

5. Las percepciones estéticas, en articular las del espacio y el tiempo, se han alterado por completo. Los antepasados no tenían ningún concepto de la "velocidad" y el movimiento tal como son percibidos hoy en día. (Bell, 1991, pp. 221 y 222). 
Otra característica de la sociedad post-industrial es que se organiza en torno al conocimiento para lograr el control social, orienta la innovación y los cambios lo que impacta en las relaciones que se establecen en la estructura social, para lo cual es necesaria que sean políticamente dirigidas. En tal sentido, Bell lo sintetiza mediante una frase contundente: «el objetivo de la nueva tecnología intelectual es, ni más ni menos, el de realizar el sueño de 'ordenar' la sociedad de masas» (Bell, 1991, p. 52).

\section{La sociedad de la información}

El sociólogo japonés Masuda presenta el concepto de Sociedad de la información para explicar el surgimiento de una nueva fase en el desarrollo de la humanidad sustentado en un nuevo paradigma: la tecnología del ordenador. Deja atrás el término Sociedad post-industrial, utilizado principalmente por el sociólogo Touraine y el economista Bell para reemplazarlo por una nueva concepción en el que la información se encuentra en el centro de las transformaciones.

En tal sentido, define a la era de la información como «el período de tiempo durante el cual tiene lugar una innovación en la tecnología de la información, la que se convierte en la fuerza latente de la transformación social, capaz de acarrear una expansión en la cantidad de información y un aumento a gran escala del almacenamiento de información» (Masuda, 1984, p. 67).

Desde su visión, el ordenador adquiere una importancia fundamental debido a que por primera vez en la historia surgió una tecnología para crear y suministrar información y cuya función principal es la de amplificar la labor mental del ser humano. «El ordenador es una máquina lógica, que ha marcado 
una época, equipada con las tres funciones de procesamiento de información: memoria, cálculo y control, que han sobrepasado capacidad humana para generar información» (Masuda, 1984, p. 68).

Por lo tanto, "la revolución de la información" va de la mano del desarrollo del ordenador debido a que posibilitará la producción masiva y sistematizada de la información, tecnología y conocimientos, a través de la conformación de bancos de datos y redes de información.

En tal sentido, el teórico japonés sostiene que el ordenador reemplazará a «la fábrica como símbolo social y se convertirá en el centro de producción y distribución de bienes de información» (1984, p. 47).

Masuda describe el surgimiento y desarrollo de la informatización a través de cuatro fases definidas: 1) a nivel científico, 2) gerencial, 3) social, 4) individual. La fase de la informatización científica, la sitúa en el período comprendido entre los años 1945-1970. Etapa en la que se comenzó a emplear extensamente el ordenador en proyectos a gran escala, tales como la defensa nacional y la exploración espacial: El Estado fue el conductor de la informatización y los Estados Unidos tomaron la delantera a través del programa Semi-automatic ground environment system (SAGE) contra el ataque de misiles de la Unión Soviética y en el Programa Apolo de exploración espacial (Masuda, 1984, pp. 54 y 55$)$.

La segunda fase, la de la informatización de la gestión empresarial, la circunscribe entre 1955 y 1980 . En este período se experimenta un traslado de la computarización desde la ciencia hacia la gestión empresarial, tanto pública como privada, debido a la creciente aplicación de la informática en áreas administrativas y empresariales. 
La tercera fase, que establece su comienzo a partir de 1970, la denomina informática en base a la sociedad, debido a que visualiza el empleo del ordenador en beneficio del conjunto social. Esta etapa deriva en la siguiente, que la llama informática basada en el individuo, que es posibilitada por la invención de los circuitos integrados. «Todo el mundo podrá utilizar información de ordenador obtenida de sistemas hombre-máquina (sistemas en los que el diálogo entre el hombre y la máquina se dará como una conversación) para resolver problemas y para perseguir las nuevas posibilidades del futuro» (Masuda, 1984, p. 57).

El teórico japonés analiza la aplicación de los avances tecnológicos motorizados en sociedades avanzadas de su época, principalmente la japonesa, la estadounidense, la sueca, entre otras, para establecer los rasgos que tendrá la sociedad global en las siguientes décadas al efectuar una proyección de las tecnologías surgidas en esas estructuras sociales.

En tal sentido vincula la concreción de la Sociedad de la información con un tipo específico de informatización muy similar al funcionamiento de internet en la actualidad.

Para ello, elaboró el concepto de "unidad productora de información", que la definió como una «infraestructura de información que consiste en diversas unidades y servicios de proceso de información pública y que combina el ordenador con las redes de comunicaciones. Desde estas unidades, cualquiera, en cualquier lugar y en cualquier momento, podrá obtener fácil y rápidamente la información que desee y a bajo costo» (Masuda, 1984, p. 94). Pero además, se animó a plantear los requisitos que debería cumplir una unidad productora de información: 
1. Unidades equipadas con ordenadores grandes, capaces de realizar un proceso paralelo simultáneo, conectados a aparatos con gran capacidad de memoria, disponiendo de un gran número de programas, así como muchas bases de datos. Estos equipos podrán procesar la información y darán servicio a un gran número de usuarios al mismo tiempo.

2. Estos servicios de proceso de información se pondrán a disposición del público en general, por lo que los ordenadores de las unidades centrales estarán conectados por medio de circuitos de comunicaciones, estando los terminales directos en las empresas, escuelas y hogares.

3. Cualquier usuario podrá llamar a las unidades productoras de información locales para obtener datos procesados e incluso podrá procesar los datos necesarios por sí mismo.

4. El costo de utilización de estos servicios deberá ser bajo, de manera que el público en general pueda utilizarlo para la resolución de sus necesidades cotidianas.

Se evidencia entonces, la vinculación necesaria que Masuda realiza entre la informatización -cuyo epicentro es el desarrollo del ordenador- con el surgimiento de un tipo de sociedad basada en la información.

Algunos de los postulados del autor japonés quedaron en un planteo futurista, no obstante, prevalece su capacidad para hacer una lectura del avance de la informatización que se asemeja notablemente al desarrollo global que tiene en la actualidad la red de redes.

Por su parte, el investigador español Manuel Castells, hacia finales de la década de 1990, también sustenta sus planteos en el surgimiento de una era de la información como motor de los cambios experimentados por las sociedades a nivel global y los vincula con una serie de procesos 
interrelacionados que dan paso a un nuevo modo de desarrollo que denomina informacionalismo.

«Una revolución tecnológica, centrada en torno a las tecnologías de la información, empezó a reconfigurar la base material de la sociedad a un ritmo acelerado. Las economías de todo el mundo se han hecho interdependientes a escala global, introduciendo una nueva forma de relación entre economía, Estado y sociedad en un sistema de geometría variable» (Castells, 1997, p. 26).

El núcleo de la transformación, que el autor sitúa en las postrimerías del siglo $\mathrm{XX}$, remite a las tecnologías del procesamiento de la información y de la comunicación, entre las que destaca a la microelectrónica, la informática (máquinas y software), las telecomunicaciones, televisión, radio, pero también a la ingeniería genética.

«Lo que caracteriza a la revolución tecnológica actual no es el carácter central del conocimiento y la información, sino la aplicación de ese conocimiento y procesamiento de la información/comunicación, en un círculo de retroalimentación acumulativo entre la innovación y sus usos» (Castells, 1997, p. 61).

Además, Castells plantea que surge un nuevo paradigma de la tecnología de la información como rasgo constitutivo de la Sociedad Red, que la percibe como una nueva forma de capitalismo erigido sobre la base de una sociedad de la información que gira en torno a nuevas estructuras organizacionales que surgen alrededor de los medios de comunicación electrónicos, y que determina una de sus principales características: su carácter global y estructurado en torno a una red de flujos financieros que necesitan basarse en el conocimiento generado y procesado por la tecnología de la información. 
En esta estructura social se destaca la convergencia creciente de tecnologías en un sistema integrado de información, que se encamina hacia una interdependencia sostenida entre las telecomunicaciones, la microelectrónica y los avances en la investigación biológica, que depende cada vez más de la mayor potencia de los ordenadores.

\section{La Cuarta revolución industrial o revolución digital}

El economista alemán Schwab, a través de su libro La cuarta revolución industrial contribuyó a instalar el concepto que se sustenta en la idea de que la tecnología y la digitalización son las impulsoras de una nueva revolución sin precedentes en la historia de la humanidad.

Los cambios experimentados por las sociedades a escala global son comparados, por numerosos autores, con la profunda transformación social impulsada por la Revolución industrial, que incluyó un cambio tecnológico, pero que principalmente derribó los cimientos de una sociedad preindustrial y obligó a generar un nuevo marco en el que las relaciones sociales, productivas e institucionales fueron modificadas de manera drástica.

Las transformaciones derivadas de la denominada Cuarta revolución industrial - Revolución digital también son percibidas como una instancia de transformación en todas las esferas sociales al provocar cambios institucionales, en los vínculos sociales y en la organización de la producción de bienes y servicios.

Más allá del desarrollo de otro tipo de tecnologías el uso social generalizado de Internet en la década de 1990 se constituyó en un factor de cambio clave, tanto que se convirtió en una tecnología omnipresente. 
En tal sentido, Internet es considerada como la estructura con la capacidad de redefinir la manera en que gran parte de las sociedades del planeta se comunican e informan, adquieren nuevos hábitos de consumo, nuevas formas de interacción que inciden en el marco de relaciones establecidas, en una forma novedosa de percibir el mundo en todas sus facetas.

Aunque muchos especialistas sostienen que este sistema tecnológico con capacidad transformadora todavía debe ser regulado para que sea seguro, viable e inclusivo, debido a que casi la mitad de los habitantes a escala mundial aún no tienen acceso a la red de redes.

Este desarrollo posibilitó y potenció la conexión entre miles de millones de personas a dispositivos móviles, que deriva en una capacidad de procesamiento y almacenamiento nunca antes alcanzado. Pero además, la Revolución digital se sustenta en los acelerados avances tecnológicos en los campos de la inteligencia artificial (IA), la robótica, el internet de las cosas (IOT), los vehículos autónomos, la impresión 3D, la nanotecnología, la biotecnología, la ciencia de materiales, el almacenamiento de energía y la computación cuántica, principalmente. (Schwab, 2016, p. 8).

Schwab, no es especialmente novedoso al proponer el concepto de Cuarta revolución industrial debido a que se sustenta, al igual que muchos de los teóricos que lo precedieron, en la capacidad de la tecnología, sumada a la digitalización, como las impulsoras de una nueva revolución sin precedentes en la historia de la humanidad por su velocidad, magnitud y alcance.

Desde una visión que pone el foco principalmente en los factores asociados al crecimiento económico, el fundador del Foro Económico Mundial sostiene que, en los albores del siglo XXI, los cambios se sustentan en la posibilidad de conexión entre miles de millones de personas a dispositivos móviles, que 
deriva en una capacidad de procesamiento y almacenamiento nunca antes alcanzado.

Al considerarla como una revolución eminentemente digital se basa en su capacidad de conectar las cosas del mundo físico (como ropa, accesorios, hogares, redes de transporte y de energía, ciudades) a las personas a través de redes y plataformas virtuales y otros tipos de tecnologías.

El signo de los nuevos tiempos lo asocia a la era digital «en la que muchas nuevas empresas proveen "bienes de información" con costos de almacenamiento, transporte y replicación que son prácticamente nulos. Algunas empresas de tecnología disruptivas parecen requerir menos trabajadores y poco capital para prosperar» (Schwab, 2016, p. 15).

Es este tiempo histórico en el que las organizaciones con la capacidad de combinar las dimensiones digital, física y biológica son las puntas de lanza de la transformación global de la industria y sus sistemas de producción, distribución y consumo.

El caso específico lo constituyen las plataformas digitales que hacen posible lo que se denomina "economía bajo demanda" (también llamado desde algunos sectores consumo colaborativo) que son utilizadas principalmente a través de teléfonos inteligentes que derivaron en formas de consumo de bienes y servicios inexistentes hasta hace más de un lustro.

El caso de Uber se convierte en un ejemplo, debido a que impacta en la experiencia de los clientes al proveerles la aplicación la ubicación del vehículo a través del dispositivo móvil, una descripción de las características del automóvil y un proceso de pago ágil, lo que evita retrasos.

Lo digital, en estos casos, impacta en un cambio drástico del modelo de negocios que a su vez origina conflictos debido a que afecta a sectores 
competidores y genera la necesidad de establecer marcos legales que regulen las especificidades de la actividad.

«Estos modelos de negocios basados en la combinación (la compañía no es propietaria de los activos y un conductor posee el vehículo y lo pone a disposición de otros) ilustran la magnitud de la disrupción que se produce cuando se utilizan activos digitales y combinaciones de plataformas digitales existentes para reorganizar las relaciones con los activos físicos, lo cual marca un notable tránsito de la propiedad al acceso» (Schwab, 2016, p. 54).

Para Schwab, si algo caracteriza a la cuarta revolución industrial es la posibilidad que les brinda a los integrantes de una sociedad adquirir productos, acceder o solicitar de manera remota a servicios de transportes terrestres y aéreos, realizar transacciones bancarias, alquilar propiedades, escuchar música o ver películas y eventos deportivos.

Internet, el teléfono inteligente e innumerable cantidad de aplicaciones son posibilitadores de nuevas formas de lectura, navegación y comunicación que impactan en las relaciones entre los individuos, pero también en el mundo del trabajo y de la producción, entre muchos otros sectores.

Estos modelos de negocios emergentes que se sustentan en el desarrollo de internet se caracterizan por estar construidos en base a una plataforma web y no poseer activos como las empresas convencionales. Los ejemplos son cada vez más numerosos, como lo demuestran Uber, que es una empresa de traslados de pasajeros que no posee vehículos; Airbnb, proveedor de alojamientos que no posee propiedades; Facebook y Google, que concentran la mayor parte de la publicidad mediática a nivel mundial y no generan contenidos informativos propios. Todo está impactando en el funcionamiento 
comercial empresarial con los consecuentes conflictos entre los diversos sectores.

Es más, Schwab percibe las consecuencias transformadoras de la revolución digital hasta en el surgimiento de las criptomonedas asociadas al desarrollo de la tecnología del blockchain que posibilita las transacciones financieras hechas con monedas digitales, entre las cuales la más popular es el bitcoin.

El desarrollo de lo digital, por lo tanto, es el eje de una revolución en la que se sostiene una transformación social sin precedentes, en la que Schwab visualiza nuevas oportunidades para la colaboración mutua de individuos e instituciones que se encamine hacia una mayor cohesión social.

\section{Conclusiones}

Sociedad del conocimiento, de la información, cuarta revolución industrial, revolución digital son conceptos que fueron planteados por los teóricos abordados desde una mirada similar, derivada desde una visión economicista que concibe a la tecnología como una herramienta esencial para el progreso social.

Machlup analizó la transformación en la producción y el empleo en los Estados Unidos de mediados del siglo XX al indagar en los sectores empresariales e industriales, privados y públicos, y definió como conocimiento a las actividades derivadas de las nuevas tecnologías como generadoras de trabajo.

A partir de ese planteo se instala con mayor fuerza la idea de que la productividad económica se encuentra directamente asociada al avance tecnológico, cuya aplicación deriva en un desarrollo social.

Bell, que concibe y explica las características de una sociedad post-industrial sustentada por la producción en el área servicios, destaca el carácter central 
del conocimiento teórico como eje organizador de la nueva tecnología en la que se sostiene el crecimiento económico.

Más allá de explicar las transformaciones sociales experimentadas por occidente puso especial énfasis en la investigación y desarrollo a través de la unión de ciencia y tecnología para el impulso de industrias vinculadas con la computadora y la electrónica. Es por eso que propone el conocimiento teórico como un recurso estratégico a través de las universidades y las instituciones vinculadas con la investigación.

La tecnología del ordenador es la base en el que se sustenta el pensamiento de Masuda, en los albores de la década de 1980, que prevé el comienzo de una época basada en el desarrollo de la tecnología de la información, a través de los bancos de datos y de las redes de informatización, como fuerza latente para la transformación social.

Castells, por su parte, al trazar los rasgos de la Sociedad Red percibe una relación de interdependencia entre economía, Estado y sociedad centrada en la revolución de las tecnologías de la información con la capacidad de reconfigurar a las sociedades a escala global.

El núcleo de esa transformación lo relaciona con las tecnologías del procesamiento de la información y la comunicación entre las que se destacan la convergencia entre la microelectrónica, la informática, las telecomunicaciones, la televisión, la radio y la ingeniería genética.

El factor clave que el alemán Schwab tiene en cuenta como factor de cambio social en la primera década del siglo XXI es la digitalización. En tal sentido, el uso social de Internet se constituyó en un agente de cambio clave debido a que redefinió la forma en que los integrantes de las sociedades se informan, interactúan, consumen, pero además, a través de ella surgieron nuevos 
modelos de negocios mediante la creación e impulso de las plataformas digitales.

La capacidad de procesamiento y almacenamiento de datos sin precedentes, el desarrollo y uso masivo de los celulares inteligentes y la llegada del 5G son impulsores de cambios acelerados relacionados con la inteligencia artificial, la impresión 3D, la nanotecnología, la biotecnología, la computación cuántica, entre otros desarrollos.

La mirada común derivada de los planteos de los autores analizados es que entienden el desarrollo de las estructuras sociales a través de la consolidación de la relación tecnología-economía. De esta estrecha vinculación deriva la idea de que la productividad económica se encuentra directamente asociada al surgimiento de nuevas tecnologías, clave para la creación de conocimiento.

Desde esta perspectiva la producción de conocimiento es entendida como una actividad económica derivada de la capacidad de la sociedad de crear tecnologías aplicadas al desarrollo social, aunque dejan de lado los factores culturales, políticos y sociales necesarios para comprender los fenómenos de la realidad en toda su magnitud y alcance.

\section{Bibliografía}

- Bell, D. El advenimiento de la sociedad post-industrial. 3era. Edición: 1991. Madrid, España: Alianza Editorial.

- Castells, M. (1997). La era de la información. Madrid, España: Editorial Alianza.

- Machlup, F. (1962). The production and distribution of knowledge in the United States [La producción y distribución del conocimiento en los 
Estados Unidos]. New Jersey, Estados Unidos: Princeton University Press.

- Masuda, Y. (1984). La sociedad informatizada como sociedad postindustrial. Madrid, España: Fundesco y Editorial Tecnos.

- Schwab, K. (2016). La cuarta revolución industrial. Recuperado de: http://40.70.207.114/documentosV2/La\%20cuarta\%20revolucion\%20ind ustrial-Klaus\%20Schwab\%20(1).pdf 